\title{
The role of echocardiography in the diagnosis and management of hypertrophic cardiomyopathy
}

Running title: Echocardiography in HCM

Authors:

Fernando Dominguez, MD 1,2,3*; fdominguezrodriguez@gmail.com

Esther González-López, MD, PhD1,2,3 * esthgonzalez@hotmail.com

Laura Padron-Barthe, $\mathrm{PhD}^{1,2,3}$; padronlaura@yahoo.fr

Miguel Angel Cavero, MD¹; mcaverog@gmail.com

Pablo Garcia-Pavia MD, PhD 1,2,3,4; pablogpavia@yahoo.es (corresponding author)

\section{Affiliation:}

${ }^{1}$ Heart Failure and Inherited Cardiac Diseases Unit. Department of Cardiology. Hospital Universitario Puerta de Hierro, Madrid, Spain.

${ }^{2}$ Myocardial Biology Programme, Centro Nacional de Investigaciones Cardiovasculares (CNIC), Madrid, Spain.

${ }^{3}$ Centro de Investigacion Biomedica en Red de Enfermedades Cardiovasculares (CIBERCV), Spain.

${ }^{4}$ Francisco de Vitoria University, Madrid, Spain.

Word count: 4,060

*Dr Dominguez and Dr Gonzalez-Lopez contributed equally as first authors

Disclosures: None.

\section{Address for Correspondence:}

Pablo Garcia-Pavia, MD, PhD

Department of Cardiology. Hospital Universitario Puerta de Hierro

Manuel de Falla, 2. Majadahonda, Madrid, 28222, Spain

Tel: +34-911917297, Fax: +34-911917718

Email: pablogpavia@yahoo.es 


\section{Learning objectives:}

- To understand the key role of echocardiography in the diagnosis and management of hypertrophic cardiomyopathy (HCM).

- To learn the fundamental features that an echocardiographic report of a HCM patient should include.

- To differentiate sarcomeric HCM from phenocopies based on echocardiographic findings.

- To understand the pros and cons of each echo modality (transthoracic (TTE), transoesophageal (TOE), 3-dimensional (3D)) in different clinical scenarios.

- To appreciate the importance of echo monitoring during invasive procedures in obstructive HCM. 


\section{Introduction}

Hypertrophic cardiomyopathy (HCM) is the most common genetic cardiovascular disease, affecting one in 500 individuals in the general population. In $60 \%$ of cases, HCM presents an autosomal dominant trait due to mutations in genes encoding cardiac sarcomeric proteins, and the remaining aetiologies include a variety of clinical entities such as glycogen and lysosomal storage disorders, mitochondrial diseases or cardiac amyloidosis, among others(1). In all cases, a systematised echocardiographic approach is essential to establish the diagnosis of HCM and to decide on specific therapeutic strategies. Moreover, echocardiography plays a key role in the evaluation and guidance of the different options to tackle obstructive HCM (HOCM). Indeed, recent improvements in survival and quality of life in HCM might be attributed to several factors, including the development of reliable imaging techniques. On top of this, echocardiography provides valuable information for the management of HCM regarding other aspects beyond left ventricular tract obstruction (LVOTO). This includes sudden cardiac death (SCD) prediction, thromboembolic risk stratification and family screening.

In this article, we review and focus on the current role of echocardiography in the diagnosis and management of HCM. 


\section{Role of echocardiography in the diagnosis and evaluation of HCM}

A systematic echocardiographic approach is essential to establish the diagnosis of HCM and should include the following features (summarised in Figure 1).

\section{Left ventricular hypertrophy evaluation}

In individuals with suspected HCM and in relatives of HCM patients, a complete evaluation of all left ventricular (LV) segments from base to apex should be performed. Measurements of LV wall thickness must be obtained at end-diastole and in short-axis views ${ }^{(2)}$. Systematic short-axis views at mitral valve level, at the base of papillary muscles and at apex are recommended (Figure 2) to allow reliable successive measurements during follow-up.

The most common LV hypertrophy (LVH) pattern in HCM is the asymmetrical thickening of the inter-ventricular septum. However, hypertrophy might be also present elsewhere in a non-contiguous manner, extending to the lateral wall, inferoseptum or apex(3). This asymmetric distribution of hypertrophy has been considered an important diagnostic feature of HCM, as other causes of LVH due to increased loading conditions, such as hypertension, tend to exhibit a more symmetrical pattern(4). Nonetheless, some elderly patients with hypertension and no history of HCM may also present an asymmetric distribution of LVH due to the presence of a ventricular septal bulge. Consequently, differential diagnosis may be challenging and other features such as a posterior wall $>11 \mathrm{~mm}$, the presence of abnormal papillary muscles, elongated mitral leaflets, systolic anterior motion (SAM) or LVOTO could help in distinguishing HCM and isolated LV septal bulge(5).

Other patterns of LVH in HCM include non-septal asymmetric hypertrophy, the apical form, and the less frequently observed symmetric and concentric pattern(2). 
When wall thickness is increased in the anterolateral wall or the apex, its measurement could be difficult and so imaging acquisition should be particularly thorough. Ultrasound contrast agents or three-dimensional (3D) echography could be helpful in this context(6,7).

Moreover, beam alignment and correct orientation are essential to prevent wall thickness (WT) overestimation or foreshortening and caution should be exercised to exclude right ventricle (RV) structures such as trabeculations and the RV moderator band(7).

Accepted two-dimensional echocardiographic HCM diagnostic criteria include:

- Unexplained maximal WT $\geq 15 \mathrm{~mm}$ (or higher than 2 standard deviations (SD) for age, gender, and height) in any myocardial segment.

- For first-degree relatives of HCM patients, an unexplained maximal WT $\geq 13 \mathrm{~mm}$ is also considered diagnostic.

Asymmetric septal hypertrophy is defined as a septal/posterior WT ratio of $>1.3$ in normotensive patients ( $>1.5$ in hypertensive patients $)^{(7)}$.

In some cases, HCM patients concurrently present RV hypertrophy. Thus, RV WT should also be evaluated. A maximal WT $\geq 5 \mathrm{~mm}$ at end-diastolic subcostal or parasternal long-axis views at tricuspid chordae level should be considered as a hypertrophied RV(7). 


\section{Systolic function}

Evaluation of systolic function can be challenging in HCM. The classical parameters such as ejection fraction (EF) or fractional shortening (FS) are usually normal or even increased due to preserved radial contractile function. However, longitudinal strain (LS) values using speckle-tracking echocardiography have been reported to be decreased in $\mathrm{HCM}$ patients despite normal $\mathrm{EF}^{(8)}$. Moreover, tissue Doppler imaging (TDI) techniques have shown decreased systolic and diastolic velocities from very early phases of the disease and also in HCM mutation carriers without overt LVH(9).

Once LVH is present, the lower LS values are found in hypertrophied segments, as opposed to normal subjects who show homogeneous strain and strain rates in all LV regions(10).

Later on in the course of the disease, 5-10\% of HCM patients develop a "burnt-out" phase in which LV dilates, WT regresses and EF decreases(11).,While the mechanism is unclear, diffuse myocardial ischaemia due to microvascular dysfunction may play a role leading to cell death and fibrosis replacement(12).

An EF below 50\% represents severe systolic dysfunction, and although it is often underappreciated, once end-stage HCM develops clinical deterioration is rapid. Therefore, the therapeutical approach must be aggressive once EF is $<50 \%{ }^{(1)}$.

\section{Diastolic function}

Diastolic dysfunction is one of the main causes of dyspnoea in patients with HCM, and a transthoracic echocardiogram (TTE) is the preferred technique for its assessment. 
Following the last American Society of Echocardiography (ASE) and the European Association of Cardiovascular Imaging (EACVI) guidelines for diastolic function assessment ${ }^{(13)}$, four criteria should be included in the evaluation:

$-\mathrm{E} / \mathrm{e}^{\prime}>14$.

- Left atrial volume index $>34 \mathrm{~mL} / \mathrm{m}^{2}$.

- Pulmonary vein atrial reversal velocity (Ar-A duration) $\geq 30 \mathrm{msec}$.

- Peak velocity of tricuspid regurgitation (TR) jet by continuous wave (CW) Doppler $>2.8 \mathrm{~m} / \mathrm{sec}$.

If two or more of these criteria are met, a diastolic dysfunction grade II or higher is present.

Other parameters such as an E/a ratio $>2$, an abnormally reduced mitral annular $\mathrm{e}^{\prime}$ velocity (septal $<7 \mathrm{~cm} / \mathrm{sec}$, lateral $<10 \mathrm{~cm} / \mathrm{sec}$ ) or a E-wave deceleration time $<150$ ms imply a restrictive LV filling, and some studies have shown a worse clinical outcome in these individuals even when EF is preserved(14).

\section{Assessment of left atrial size}

The left atrium (LA) is often enlarged in HCM patients due to mitral regurgitation (MR) and high LV filling pressures.

Anteroposterior (AP) diameter has been widely used in HCM and has been proven useful to predict embolic events, sudden cardiac death and long-term prognosis in

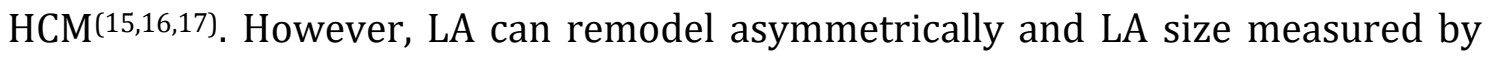
AP diameter may not always be accurate. Left atrial volume indexed to body surface reflects more precisely LA dimension and is the recommended parameter to evaluate LA size by the ASE/EACVI because it considers all spatial dimensions ${ }^{(18)}$. 
Assessment of the mitral valve and left ventricular outflow tract obstruction

The MV apparatus should be systematically evaluated in HCM, including leaflets, chordae and papillary muscles.

Most patients with obstructive HCM present elongated anterior and posterior mitral leaflets. The average length of the anterior leaflet in these patients is $34 \mathrm{~mm}$ (compared with $24 \mathrm{~mm}$ in normal subjects), leading to an extent into the LV cavity above the mitral annulus plane (a mean of $26 \mathrm{~mm}$ vs $13 \mathrm{~mm}$ in controls)(19). Other MV abnormalities typically present in HCM patients are listed in Table $1^{(7,19) .}$

Anomalous chordae insertion may tent the mitral leaflet anteriorly towards the flow stream, contributing to SAM, which is responsible for LVOTO. Likewise, displaced papillary muscles contacting the septum and elongated mitral leaflets are implicated in the pathophysiology of LVOTO(19).

However, SAM is not exclusive of $\mathrm{HCM}$ as it can appear in the context of hypovolaemia, in hypertensive patients with small ventricles, with inotropes or after MV surgery repair(20). Incomplete SAM is considered when the anterior mitral leaflet fails to contact the IVS and is considered complete when it contacts the IVS. The severity of SAM depends on how long the mitral leaflets contact the IVS during systole. Exercise echo can also aid in the assessment of the dynamic component. LVOTO, defined as a peak gradient $>30 \mathrm{mmHg}$, is present at rest in one third of HCM patients and should be quantified from the apical 5- and 3-chamber view using continuous wave (CW) Doppler. LVOTO occurs because of interventricular septal (IVS) hypertrophy and narrowing of the LVOT, as well as SAM of the mitral valve (MV). Although the threshold of $30 \mathrm{mmHg}$ defines HOCM, it is generally 
accepted that obstruction becomes haemodynamically relevant when it is $\geq 50$ $\operatorname{mmHg}(1)$.

LVOTO can be present at rest or after provocative manoeuvres such as standing, Valsalva or exercise, which uncovers a third of HOCM patients with no obstruction at $\operatorname{rest}^{(21)}$ (Figure 3). In the case of patients with a gradient $<50 \mathrm{mmHg}$ at rest or with bedside manoeuvres but still symptomatic, an exercise echocardiogram is mandatory to unmask latent obstruction(21). By contrast, dobutamine pharmacological stress echocardiogram is not recommended as it can be poorly tolerated due to its $\beta 2$ vasodilator effect. Regarding nitrates, current ESC guidelines still reserve echocardiogram with these drugs to those subjects who cannot perform physiologically stressful procedures(1).

LVOT gradient can be difficult to acquire, especially during stress echocardiograms, and it must not be mistaken for the MR jet (Figure 4).

Regarding the RV, outflow obstruction can also be present, isolated or combined with LVOTO. It is usually caused by the mid-systolic contact of prominent right ventricular muscle bundles located in the RVOT. As it can be easily missed, it should be specifically evaluated, especially in young HCM patients with massive hypertrophy

\section{Left ventricular mid-cavity obstruction}

Patients without LVOTO can still present mid-cavity obstruction, which accounts for $10 \%$ of HCM cases. Subjects with mid-ventricular obstruction are usually very symptomatic and do not respond to drugs such as beta-blockers, calcium channel blockers or disopyramide. Mid-cavity LV hypertrophy with a normal apical 
thickness and anomalous papillary muscle insertion are responsible for this phenotype, which is commonly associated with apical aneurysms (Video S1 Supplementary Material) and shows a paradoxical apex to base diastolic gradient and an aliasing in the sequestered apical area.

\section{Differential diagnosis}

Hypertensive heart disease and athlete's heart are two situations in which HCM differential diagnosis could be considered. Several morphological features that can be evaluated with echocardiography can help to distinguish these entities (Table 2). Furthermore, ECG and family history are always necessary to contextualise the echocardiographic findings. For instance, patients with hypertensive heart disease or athlete's heart can fulfil LVH criteria on ECG, but repolarisation abnormalities are not as common as in HCM.

Another important group of diseases to be considered in the differential diagnosis of HCM are the so called phenocopies, or non-sarcomeric variants of HCM. These include a variety of clinical entities such as glycogen and lysosomal storage disorders, mitochondrial diseases, disorders of fatty acid metabolism and cardiac amyloidosis $(\mathrm{CA})^{(22,23)}$. In this context, some echocardiographic features are important diagnostic red flags (Table 2, Figure 5).

Regarding CA, recent data suggest that this disease could be extremely frequent (24) and it was reported recently that $5 \%$ of $\mathrm{HCM}$ patients have hereditary transthyretin $\mathrm{CA}^{(25)}$. Typical features of $\mathrm{CA}$ include a granular myocardial texture, mild pericardial effusion, thickening of the interatrial septum and atrioventricular valves and a restrictive phenotype, as well as reduced EF in advanced stages(22). Moreover, a relative apical sparing of longitudinal strain using 2D speckle-tracking 
echocardiography has been described in CA patients, as opposed to patients with other forms of LVH including sarcomeric $\mathrm{HCM}^{(26)}$ (Figure 6).

\section{Echocardiography in HCM management}

Echocardiography plays a decisive role in several aspects of HCM management. Areas where echocardiography is decisive include: LVOTO management (procedure selection and guidance), SCD and embolic risk prediction, risk stratification in non-cardiac surgery, and familial screening.

\section{LVOTO management}

Preprocedurural evaluation and procedure selection

Echocardiography has classically played a crucial role in LVOTO management. It was demonstrated in 1971 that SAM is abolished by myectomy(27). Moreover, echocardiography has helped to mechanistically understand LVOTO and has contributed to the evolution of myectomy from Morrow's procedure to the current extended technique(27).

When considering a patient for myectomy or alcohol septal ablation (ASA), a complete preprocedural echo study should address, firstly, the mechanism of obstruction, secondly, the extension of hypertrophy and thirdly, a full assessment of MV. Table 3 includes a list of the main features and measurements that should be included(1).

Recently, 3D echocardiography has shown its potential in the preoperative scenario. 3D echocardiography can enhance the spatial assessment of the site, the septal thickness and systolic mitral leaflet-to-septal contact extent, as well as the 
aorto-septal angle(21,28,29). Nonetheless, its value in the clinical arena is still unknown and it remains predominantly a research tool(29).

ACCF/AHA guidelines favour myectomy over ASA as first-line therapy in the invasive treatment of LVOTO(30,31). By contrast, ESC HCM guidelines consider myectomy and ASA as equal procedures although advocate for a pre-interventional evaluation to decide(1).

Echocardiography can contribute to the procedure election process. When the LVOTO mechanism is unclear, pre-operative imaging is suboptimal, intrinsic severe MR is suspected, or to better assess the MV apparatus, transoesophageal echocardiogram (TOE) must be performed(1,31) (Figure 7).

It has been suggested that a minimum septal thickness $\geq 17 \mathrm{~mm}$ is necessary to consider a patient for LVOTO invasive techniques, but several referral centres accept patients with a thinner septal thickness ${ }^{(32)}$. ASA may be less effective when LVH is severe $(\geq 30 \mathrm{~mm})$ and in those cases it is generally discouraged(1,29). Additionally, MV abnormalities cannot be corrected by ASA and if present they prompt the election towards myectomy(17,33). In patients with mitral leaflet elongation and/or at least moderate MR, different repairing MV procedures can be combined at the same time as myectomy(19). When anterior leaflet measures $>33$ $\mathrm{mm}$, plication could be considered, particularly if LVH is $<20 \mathrm{~mm}$. (Figure $8 \mathrm{~A}$ ). MR caused by SAM is classically directed posteriorly and laterally and peaks in mid-to-late systole. If regurgitation is anteromedially or centrally-directed or the peak lasts throughout systole, the mitral apparatus should be examined with particular care and intrinsic MV disease should be ruled out ${ }^{(1,17,19)}$ (Figure 8B). 3Decho and TOE could be useful when the aetiology and mechanism remains unclear(1) (Video S2). 
Up to $10-20 \%$ of patients with HCM have significant MR, independent of SAM, including degenerative, rheumatic or congenital aetiologies. It is fundamental to differentiate these patients as they would benefit from MV replacement or repair (Video S3).

\section{Echocardiography during myectomy}

Imaging is an integral part of the periprocedural assessment of patients who undergo myectomy ${ }^{(34)}$. As direct cardiac visualisation could lead to imprecision in the extent of myectomy, intraoperative TOE has become an essential part of the procedure and should always be available during the intervention. TOE provides a guide to the morphology of the septum and the geometry of the LVOT, individualising the resection(19), and has been shown to increase the safety and the efficacy of the procedure(19,35).

Furthermore, intraoperative TOE contributes to surgical planning, evaluates the success of resection and MV repair and detects surgical complications (Figures 5 and 6). Intraoperative TOE can provide an immediate assessment after weaning from bypass pump(7) and can recognise suboptimal surgical results by residual obstruction or more than mild MR.

Taking into account the haemodynamic conditions under general anaesthesia during the procedure, isoproterenol administration or premature ventricular contraction (PVC) evaluating post-extraystolic potentiation (Brockenbrough manoeuvre), could be considered to assess an adequate resection(36). Gradients $>25$ mmHg or post-PVC $>50 \mathrm{mmHg}$ as well as more than mild MR should lead to revision and further resection or MV repair if appropriate(36).

TOE can also detect complications such as ventricular septal defects (VSD) and aortic regurgitation (both appear in $<1 \%$ of cases) at an early phase $e^{(1,7,33)}$. 
Postoperative echocardiographic evaluation should confirm the reduction of LVOT gradient, a thinner septum compared with the basal echo, an enlarged LVOT area, and absence of SAM and the mitral-septal contact.

The role of 3D TOE in myectomy is currently mostly unexplored but one study has shown a good performance in quantifying the volume and mass of the muscle resected $^{(7,29)}$ (Video S2).

\section{Echocardiography and ASA}

Myocardial contrast echocardiography (MCE), which is based on selective intracoronary contrast injection, has become an integral component of ASA and it is recommended in all patients undergoing ASA. The introduction of MCE has been proven to increase the possibility of success and to influence the interventional strategy in up to $20 \%$ of cases, through changing the selected vessel or cancelling the procedure(37). Among other advantages, MCE enables a reduction of ethanol and fluoroscopy time, the infarct size and the incidence of complications(34).

In MCE, echo contrast or agitated X-ray contrast is injected through the inflated balloon catheter(37). Echo shows the opacified myocardium, delineating the area of perfusion. This area has to be adjacent to and extend beyond the point at which the anterior leaflet of the MV contacts the septum and where the maximal flow acceleration concurs(37) (Video S4). MCE typically increases echo density in a delineated area of the basal septum(7) (Video S5).

The territory supplied by the perforators can be very variable and can include the moderator band, papillary muscles, the distal septum, the lateral wall and even the $\mathrm{RV}$ wall(33,34). Before alcohol is injected, absence of opacifying remote territories 
should be checked. If contrast cannot be localised exclusively at the basal septum, the procedure should be cancelled.

In most centres, MCE is performed by TTE. Multiple views including parasternal long-axis and short-axis as well as 4- and 3-chamber apical views can be used to delimit opacification of target and non-target regions. The main limitations of TTE include the difficulty of continuous monitoring and the suboptimal quality images in supine position(7,34).

For these reasons, TOE is an alternative. It provides better images although the required sedation/general anaesthesia can alter the loading conditions and interfere with LVOT gradient evaluation(7).

Intraprocedural echo is useful for evaluating the results. The basal septum, infarcted by alcohol, shows typically an increased echogenicity, decreased thickening and excursion(7,34). When MR is due to SAM, usually a reduction or elimination can be also seen(7) (Video S5).

Regarding LVOTO, many patients demonstrate a triphasic haemodynamic response. Firstly, an immediate marked decrease due to myocardial stunning or oedema appears (Figure 9); secondly, a recovery up to $50 \%$ of the pre-procedure gradient and finally, a progressive reduction over weeks to months due to scarring and thinning. Definitive results can take up to several months(37).

Procedure complications that should be monitored include VSD, cardiac tamponade and remote myocardial infarction due to collateral circulation(33,37). 


\section{Echocardiography in patients with mild hypertrophy}

Some patients present mild septal thickness $(16 \mathrm{~mm}$ at the point of the mitral leaflet septal contact) and are therefore not suitable for classic myectomy or ASA due to the risk of iatrogenic $\operatorname{VSD}(1,30)$.

In these cases, inserting a dual-chamber pacemaker (PM) is an option and biventricular pacing has also shown promising results in preliminary studies ${ }^{(38)}$ with a randomised controlled trial under way (NCT01614717).

Echo is rarely required for PM implantation. Nonetheless, after the procedure, TTE could be useful to evaluate the reduction in LVOTO. More recently, TTE has gained relevance in this setting to optimise biventricular pacing. Echo can show the best AV delay based on LVOT gradient and transmitral flow(7,34) (Figure 10).

Another alternative in these patients is cardiac surgery but swapping the goal from septal thickness to abnormalities of the MV and papillary muscles that favour $\operatorname{SAM}^{(31)}$.

Catheter-based procedures have also been proposed for this subgroup of patients. A successful experience with MitraClip ${ }^{\circledR}$ has been recently reported, shifting the residual anterior leaflet edge away from the LVOT by combining it with the posterior leaflet (A2 to P2) ${ }^{(39)}$. In this case, TOE during the procedure is essential for lining up the clip perpendicular to the leaftlet coaptation plane(38).

\section{SCD stratification}

Several parameters currently used to predict SCD are obtained from echo images(40). Maximal WT has long been considered a key aspect of SCD risk stratification and is included in all stratification scores $(1,15,30,40)$. Different studies 
reported an increased risk of SCD in patients with a maximal WT of $\geq 30 \mathrm{~mm}$, although few data are available with extreme LVH $(\geq 35 \mathrm{~mm})^{(41)}$ and a paradoxical reduction in SCD risk has been reported recently in individuals with $\mathrm{LVH} \geq 35$ $\mathrm{mm}^{(42)}$.

Other proposed factors associated with SCD derived from echo examination are LVOTO, LA dilatation, LV aneurysm, LV systolic dysfunction and low TDI velocities $(30,40,41)$.

Almost half of the predictor variables of the ESC SCD stratification risk model ${ }^{(1,16)}$ derive from echo:

- Maximal WT measured at the parasternal short-axis at any level.

- LA diameter.

- LVOTO at rest and Valsalva.

LA volume is probably a more accurate measurement than LA diameter, but no data are available on the association between SCD and LA area and volume.

Despite the fact that blood pressure response has been displaced from the new SCD score(1), exercise echocardiography may still have a role in SCD stratification. Two recent studies have highlighted its role in exercise capacity evaluation and exercise-induced wall motion abnormalities as predictors for adverse outcomes(33,43).

\section{Thromboembolic risk}

Patients with HCM face an increased risk of AF and its consequential thromboembolic risk, which affects almost $20 \%$ of these subjects(1). The widely used $\mathrm{CHA}_{2} \mathrm{DS}_{2}$-VASc score appears to have a low predictive accuracy in this subgroup of patients and therefore it is not recommended(15). 
An alternative model of thromboembolic risk quantification for HCM has recently been proposed(15), in which, AP LA diameter and MWT were independent predictors of thromboembolism(15).

A positive linear relationship between LA diameter and thromboembolic risk at 5 years was found up to a diameter of $45-50 \mathrm{~mm}$. Over $50 \mathrm{~mm}$, the risk became exponential and the prevalence of thromboembolic events when these patients were in sinus rhythm was $4.7 \%{ }^{(15)}$. Based on these data, it is common practice in our unit to initiate anticoagulation in HCM patients in sinus rhythm when LA diameter is $>50 \mathrm{~mm}$ and bleeding risk is low, while ESC guidelines recommend screening HCM patients for AF with Holter-ECG every 6-12 months when LA diameter is $>45 \mathrm{~mm}^{(1)}$.

\section{Non-cardiac surgery management}

Although HCM patients undergoing high-risk and intermediate-risk non-cardiac surgeries have a low perioperative event rate, they have a higher risk of complications than patients without $\operatorname{HCM}^{(44)}$.

Echocardiography constitutes a useful tool to provide accurate preoperative risk stratification(36) and should evaluate:

- LV systolic function.

- Degree of diastolic dysfunction.

- LVOT gradient and results of prior interventions.

- MR and abnormalities of MV and subvalvular apparatus.

- Pulmonary hypertension. 
Some of these findings could modify surgical management as the presence of MR may lead to consider endocarditis prophylaxis(45) and LVOTO could cause acquired von Willebrand disease and highlight the need for a more careful coagulation approach.

In high-risk patients, echo monitoring during surgery is desirable. TOE could be useful to determine the haemodynamic status, the presence of significant LVOTO or SAM, LV filling pressures and LV systolic dysfunction(36).

\section{Familial screening}

Echocardiography is the most widely used and practical technique for HCM familial screening. Moreover, advances in genetics have led to the identification of a large number of genotype-positive/phenotype-negative individuals. Echocardiographic studies in these individuals have revealed several parameters that are more commonly found despite the absence of overt LVH, justifying a closer follow up to detect LVH development. In this sense, TDI has demonstrated a reduction in systolic and diastolic velocities and speckle-tracking studies have shown impaired regional longitudinal strain as well as apical rotation in genetic carriers $^{(7,34,46,47)}$ Other pre-hypertrophic features include ${ }^{(34,48)}$ :

- Abnormalities in the MV apparatus such as elongated mitral leaflets.

- Papillary muscle hypertrophy.

- False tendons running parallel to the septum.

- Myocardial crypts 


\section{Echocardiography versus cardiac magnetic resonance (CMR) in the diagnosis and management of hypertrophic cardiomyopathy}

ESC guidelines recommend CMR in patients with suspected HCM who have inadequate echocardiographic windows, in order to confirm HCM diagnosis(1). Moreover, it could also be considered in patients who meet HCM criteria, in order to evaluate cardiac anatomy and determine the presence and extent of myocardial fibrosis(1). Table 4 summarises the pros and cons of CMR and echocardiography in HCM diagnosis and management.

\section{Conclusions}

Echocardiography plays an essential role in the diagnosis and management of HCM. Aside from confirming LVH, it provides very valuable information that will determine the patients' treatment in many ways, from SCD and embolic risk stratification to family screening or LVOTO treatment. Regarding this last issue, the invasive treatment of HCM goes beyond classical myectomy or ASA, as patients with HCM frequently present mitral valve abnormities that could require repair or replacement and a select group of subjects present mid or apical left ventricular obstruction and could benefit from an extended myectomy beyond the LVOT. Finally, echocardiography has even proven to be useful in pre-hypertrophic gene mutation carriers who present specific TDI, speckle-tracking and morphologic features and require a closer follow-up to monitor LVH development. 


\section{Key messages:}

- Transthoracic echocardiography is an essential and very accessible tool for HCM diagnosis, and a systematised approach is required to assess both morphological and functional specific features.

- When assessing left ventricular hypertrophy, other common clinical entities such as hypertensive heart disease or athlete's heart should be discarded. An asymmetric pattern of hypertrophy or concomitant LVOTO or SAM can be helpful in this context.

- Differential diagnosis of sarcomeric HCM and the so-called phenocopies can be challenging. Classic echocardiographic findings plus new echocardiographic technologies such as 2D speckle-tracking are useful to identify phenocopies.

- Echocardiography is very useful when choosing the most appropriate invasive approach to treat LVOTO and to assist during procedures. During myectomy, intraoperative TOE monitors myocardial resection and MV repair. Regarding ASA, myocardial contrast echocardiography is recommended to delineate the perfusion area and guide the procedure.

- Mitral regurgitation caused by SAM classically presents a postero-lateral jet and peaks in mid-to-late systole. When different features are present, the mitral valve apparatus should be systematically examined in order to discard intrinsic valve disease. 
- Echocardiography is helpful to initiate thromboembolic prophylaxis in $\mathrm{HCM}$, as the anterior posterior LA diameter measured in parasternal longaxis view is an independent predictor of stroke and systemic embolism.

- Family screening is essential in HCM patients and echocardiography is an easily available and very useful technique in this context. Aside from diagnosing new HCM cases, it also detects pre-hypertrophic features in gene mutation carriers, such as papillary muscle or MV abnormalities or decreased longitudinal strain values.

\section{Acknowledgements}

We gratefully acknowledge all the personnel from the Cardiac Imaging Unit of the Hospital Universitario Puerta de Hierro for their daily cooperation and continuous support. 


\section{References}

1. Elliott PM, Anastasakis A, Borger MA et al. 2014 ESC Guidelines on diagnosis and management of hypertrophic cardiomyopathy: The Task Force for the Diagnosis and Management of Hypertrophic Cardiomyopathy of the European Society of Cardiology (ESC). Eur Heart J. 2014;35:2733-79.

2. Maron BJ. Hypertrophic cardiomyopathy: a systematic review. JAMA. $2002 ; 287: 1308-20$.

3. Klues H.G, A. Schiffers, Maron BJ. Phenotypic spectrum and patterns of left ventricular hypertrophy in hypertrophic cardiomyopathy: morphologic observations and significance as assessed by two-dimensional echocardiography in 600 patients. J Am Coll Cardiol. 1995;26:1699-708.

4. Keller H, Wanger KC, Goepfrich M et al. Morphological quantification and differentiation of left ventricular hypertrophy in hypertrophic cardiomyopathy and hypertensive heart disease. A two dimensional echocardiographic study. Eur Heart J. 1990;11:65-74.

5. Canepa M, Pozios I, Vianello PF et al. Distinguishing ventricular septal bulge versus hypertrophic cardiomyopathy in the elderly. Heart 2016;102:108794.

6. Senior R, Becher H, Monaghan M et al.Contrast echocardiography: evidencebased recommendations by European Association of Echocardiography. Eur J Echocardiogr 2009;10:194-212.

7. Cardim N, Galderisi M, Edvardsen $\mathrm{T}$ et al. Role of multimodality cardiac imaging in the management of patients with hypertrophic cardiomyopathy: an expert consensus of the European Association of Cardiovascular Imaging Eur Heart J Cardiovasc Imaging 2015;16:280. 
8. Serri K, Reant P, Lafitte $\mathrm{M}$ et al. Global and regional myocardial function quantification by two-dimensional strain: application in hypertrophic cardiomyopathy. J Am Coll Cardiol 2006;47:1175-81

9. Nagueh SF, McFalls J, Meyer D et al. Tissue Doppler imaging consistently detects myocardial abnormalities in patients with hypertrophic cardiomyopathy and provides a novel means for an early diagnosis before and independently of hypertrophy. Circulation. 2001;104:128-30.

10. Yang H, Carasso S, Woo A et al. Hypertrophy pattern and regional myocardial mechanics are related in septal and apical hypertrophic cardiomyopathy. J Am Soc Echocardiogr. 2010;23:1081-9.

11. Garcia-Pavia P, Vázquez ME, Segovia J, et al. Genetic basis of end-stage Hypertrophic Cardiomyopathy. Eur J Heart Fail. 2011;13:1193-201.

12. Galati G, Leone O, Pasquale, et al. Histological and Histometric Characterization of Myocardial Fibrosis in End-Stage Hypertrophic Cardiomyopathy: A Clinical-Pathological Study of 30 Explanted Hearts. Circ Heart Fail. 2016 Sep 12;9(9):e003090.

13. Nagueh SF, Smiseth OA, Appleton CP et al. Recommendations for the Evaluation of Left Ventricular Diastolic Function by Echocardiography: An Update from the American Society of Echocardiography and the European Association of Cardiovascular Imaging. Eur Heart J Cardiovasc Imaging. 2016; $17: 1321-60$

14. Biagini E, Spirito P, Rocchi G et al. Prognostic implications of the Doppler restrictive filling pattern in hypertrophic cardiomyopathy. Am J Cardiol. 2009;104:1727-31. 
15. Guttmann, OP, Pavlou M, Ambler G et al. Prediction of thrombo-embolic risk in patients with hypertrophic cardiomyopathy (HCM Risk-CVA). Eur J Heart Fail. 2015;17:837-45.

16. O`Mahony C, Jichi F, Pavlou M et al. A novel clinical risk prediction model for suden cardiac death in hypertrophic cardiomyopathy (HCM risk-SCD). Eur Heart J. 2014;35:2010-20.

17. Pantazis A, Vischer AS, Perez-Tome MC, Castelletti S. Diagnosis and management of hypertrophic cardiomyopathy. Echo Res Pract. 2015;2:R4553.

18. Lang RM, Berdano LP, Mor-Avi V, et al. Recommendations for cardiac chamber quantification by echocardiography in adults: an update from the American Society of Echocardiography and the European Association of Cardiovascular Imaging. J Am Soc Echocardiogr. 2015;28:1-39 e14.

19. Sherrid MV, Balaram S, Kim B et al. The Mitral Valve in Obstructive Hypertrophic Cardiomyopathy: A Test in Context. J Am Coll Cardiol. 2016;67:1846-58.

20. Nagueh SF, Bierig SM, Budoff MF et al. American Society of Echocardiography clinical recommendations for multimodality cardiovascular imaging of patients with hypertrophic cardiomyopathy. J Am Soc Echocardiogr. 2011;24:473-98.

21. Shah JS, Esteban MT, Thaman R et al. Prevalence of exercise-induced left ventricular outflow tract obstruction in symptomatic patients with nonobstructive hypertrophic cardiomyopathy. Heart. 2008;94:1288-94.

22. Rapezzi C, Arbustini E, Caforio AL et al. Diagnostic work-up in cardiomyopathies: bridging the gap between clinical phenotypes and final 
diagnosis. A position statement from the ESC Working Group on Myocardial and Pericardial Diseases. Eur Heart J. 2013;34:1448-58.

23. Barriales-Villa R, Gimeno-Blanes JR, Zorio-Grima E, et al. Plan of Action for Inherited Cardiovascular Diseases: Synthesis of Recommendations and Action Algorithms. Rev Esp Cardiol (Engl Ed). 2016;69:300-9.

24. Gonzalez-Lopez E, Gallego-Delgado M, Guzzo-Merello G, det al. Wild-type transthyretin amyloidosis as a cause of heart failure with preserved ejection fraction. Eur Heart J 2015;36:2585-94.

25. Damy T, Costes B, Hagège AA et al. Prevalence and clinical phenotype of hereditary transthyretin amyloid cardiomyopathy in patients with increased left ventricular wall thickness. Eur Heart J. 2016;37:1826-34

26. Phelan D, Collier P, Thavendirananathan et al. Relative apical sparing of longitudinal strain using two-dimensional speckle-tracking echocardiography is both sensitive and specific for the diagnosis of cardiac amyloidosis. Heart. 2012;98:1442-8.

27. Maron BJ, Maron MS. The remarkable 50 years of imaging in HCM and how it has changed diagnosis and management: From M-Mode echocardiography to CMR. JACC Cardiovasc Imaging. 2016;9:858-72.

28. Nash PJ, Agler DA, Shin JH et al. Images in cardiovascular medicine. Epicardial real-time-3-dimensional echcardiography during septal myectomy for obstructive hypertrophic cardiomyopathy. Circulation. 2003;108:e54-5.

29. Sadat K, Diddi HP, Klas B et al. Live/real time three-dimensional transesophageal echocardiographic assessment of ventricular septal 
volume and mass before and after myectomy in hypertrophic cardiomyopathy. Echocardiograhy. 2013;30:1227-31.

30. Gersh BJ, Maron BJ, Bonow RO et al. 2011 ACCF/AHA guideline for the diagnosis and treatment of hypertrophic cardiomyopathy: a report of the American College of Cardiology Foundation/American Heart Association Task Force on Practice Guidelines. J Am Coll Cardiol. 2011;58:e212-60.

31. Sen-Chowdhry S, Jacoby D, Moon JC, McKenna WJ. Update on hypertrophic cardiomyopathyy and a guide to the guidelines. Nat Rev Cardiol. 2016;13:651-675.

32. Ferrazzi P, Spirito P, Iacovoni A et al. Transaortic Chordal Cutting: Mitral valve repair fod obstructive hypertrophic cardiomyopathy with mild septal hypertrophy. J Am Coll Cardiol. 2015; 66: 1687-96

33. Patil PV, Wiegers SE. Echocardiography for hypertrophic cardiomyopathy. Prog Cardiovasc Dis. 2014;57:91-9.

34. Weissler-Snir A, Crean A, Rakowski H. The role of imaging in the diagnosis and management of hypertrophic cardiomyopathy. Expert Rev Cardiovasc Ther. 2016;14:51-74.

35. Marwick TH, Stewart WJ, Lever HM et al. Benefits of intraoperatove echocardiography in the surgical management of hypertrophic cardiomyopathy. J Am Coll Cardiol. 1992;20:1066-72.

36. Hensley N, Dietrich J, Nyhan D et al. Hypertrophic cardiomyopathy: a review. Anesth Analg. 2015;120:554-69.

37. Fifer M.A, Sigwart U. Hypertrophic obstructive cardiomyopathy: Alcohol septal ablation. Eur Heart J 2011;32:1059-1064. 
38. Berruezo A, Vatasescu R, Mont L et al. Biventricular pacing in hypertrophic obstructive cardiomyopathy: a pilot study. Heart Rhythm. 2011;8:221-7.

39. Sorajja P, Pedersen WA, Bae R et al. First experience with percutaneous mitral valve plication as primary therapy for symptomatic obstructive hypertrophic cardiomyopathy. J Am Coll Cardiol. 2016;67:2811-8.

40. Geske JB, Ommen SR. Role of imaging in evaluation of sudden cardiac death risk in hypertrophic cardiomyopathy. Curr Opin Cardiol. 2015;30:493-9.

41. Christiaans I, van Engelen K, van Langen IM et al. Risk stratification for sudden cardiac death in hypertrophic cardiomyopathy: systematic review of clinical risk markers. Europace. 2010;12:313-21.

42. O'Mahony C, Jichi F, Monserrat L et al. Inverted U-Shaped Relation Between the Risk of Sudden Cardiac Death and Maximal Left Ventricular Wall Thickness in Hypertrophic Cardiomyopathy. Circ Arrhythm Electrophysiol. 2016;9. pii: e003818. doi: 10.1161/CIRCEP.115.003818.

43. Williams LK, Gruner $\mathrm{CH}$, Rakowski $\mathrm{H}$. The role of echocardiography in hypertrophic cardiomyopathy. Curr Cardiol Rep. 2015;17:6.

44. Dhillon A, Khana A, Randhawa MS et al. Perioperative outcomes of patients with hypertrophic cardiomyopathy undergoing non-cardiac surgery. Heart. $2016 ; 102: 1627-32$.

45. Dominguez F, Ramos A, Bouza E et al. Infective endocarditis in hypertrophic cardiomyopathy: A multicenter, prospective, cohort study. Medicine (Baltimore). 2016; 95:e4008. doi: 10.1097/MD.0000000000004008.

46. Shetty R, Samanth J, Nayak K et al. Evaluation of subtle left ventricular systolic abnormalities in adult patients with hypertrophic cardiomyopathy. J Clin Diagn Res. 2014; 8: MC05-9. 
47. Michels, M, Soliman OI, Kofflard MJ et al. Diastolic abnormalities as the first feature of hypertrophic cardiomyopathy in Dutch myosin-binding protein C founder mutations. JACC Cardiovasc Imaging. 2009; 2: 58-64.

48. Maron BJ, Ommen SR, Semsarian C et al. Hypertrophic cardiomyopathy: present and future, with translation into contemporary cardiovascular medicine. J Am Coll Cardiol. 2014;64:83-99. 


\section{Figures}

Figure 1. Echocardiographic assessment of HCM: key points.

Figure 2. Left ventricular hypertrophy evaluation in HCM. Short axis views (SAX) at mitral valve level (left), mid ventricular level (centre) and apex (right). The red arrow indicates maximal wall thickness (MWT) at the apical level.

Figure 3. Exercise echocardiography. Apical 5-chamber view at rest (A) and during exercise (C). Continuous Doppler (CW) at the LVOT with a maximum pressure gradient of $11 \mathrm{mmHg}$ at rest (B), increasing up to $104 \mathrm{mmHg}$ with exercise (D).

Figure 4. CW at the LVOT showing a dynamic obstruction due to HCM. This curve is typically "dagger" shaped and presents a late peaking velocity (shaded red). Caution must be taken to avoid contamination by the mitral regurgitation or aortic stenosis jets (shaded blue).

Figure 5. Parasternal long axis views of 4 patients with different diseases that cause LVH.

A. Sarcomeric HCM with an asymmetric septal LVH. B. Anderson-Fabry disease: thickened mitral valve and a relative thinning of the inferolateral wall. C. Danon's disease with a severe concentric LVH. D. Wild-type TTR amyloidosis: Concentric LVH, large left atrium and a granular/sparkly myocardium pattern with mild pericardial effusion and thickened mitral and aortic valves. 
Figure 6. Example of longitudinal strain “bull's eye” plot . A relative apical sparing is present with lower LS values in basal and mid-ventricular segments.

Figure 7. Intraoperative transoesophageal echocardiogram (TOE) in a patient with HOCM undergoing myectomy.

A. Longitudinal view (Aortic valve level, $130^{\circ}$ ). Measurement of maximal wall thickness. B. Longitudinal view. Distance from the distal narrowing point to the right coronary cusp (RCC). C. Longitudinal view with colour Doppler. Distance from the point of anterior mitral valve leaflet contact with the septum to RCC. D. Transgastric view. Peak LVOT gradient.

Figure 8. Mitral valve and mitral regurgitation (MR) intraoperative evaluation.

A. Longitudinal view from an intraoperative TOE showing anterior mitral leaflet measurement and calcified annulus. B. Colour Doppler showing moderate-tosevere central MR in the context of intrinsic valve disease.

\section{Figure 9. Basal LVOT gradient and haemodynamic response to ASA.}

A. Basal transthoraric Doppler showing LVOTO.

B. Myocardial contrast echocardiography delineating the target area in the basal septum, with absence of opacifying in remote territories.

C. Myocardial contrast echocardiography showing increased echogenicity in the basal septum, once the alcohol is infused. 
D. LVOT gradient after alcohol injection in a branch from the first septal perforator vessel.

Figure 10. Transthoraric echocardiography optimisation in a HOCM patient with resynchronisation therapy.

A. AV interval optimisation. Final AV of $80 \mathrm{~ms}$ with $47 \%$ filling of RR interval and without A wave truncation. B. VV interval optimisation. From left to right: 1.) LVOT gradient in basal echo before CRT-P implantation. 2.) Resting LVOT gradient with + 30 VV interval. 3.) Vasalva LVOT gradient with + 30 VV interval. 4.) Final VV with Valsalva gradient of $43 \mathrm{mmHg}$. 


\section{Tables}

Table 1. Mitral valve abnormalities in HCM

\begin{tabular}{|l|l|}
\hline \multicolumn{2}{|c|}{ Mitral valve abnormalities in HCM } \\
\hline Leaflet abnormalities & - Excessive tissue and elongated leaflets. \\
& - Prolapse. \\
& - Leaflet mismatch. \\
& - Calcification. \\
\hline Chordae abnormalities & - Anomalous insertion. \\
& - Fibrotic retraction. \\
\hline Papillary muscles abnormalities & - Hypertrophied. \\
& - Anterior and basilar displacement of the \\
& anterolateral papillary muscle. \\
& - Insertion of anterolateral papillary muscle \\
& directly into the mid-anterior mitral valve leaflet. \\
\hline Annulus & - Calcification. \\
\hline
\end{tabular}


Table 2. Echocardiographic differential diagnosis in patients with hypertrophied left ventricles

\begin{tabular}{|l|l|l|l|l|l|}
\hline & $\begin{array}{l}\text { Hypertrophic } \\
\text { cardiomyopathy }\end{array}$ & $\begin{array}{l}\text { Hypertensive heart } \\
\text { disease }\end{array}$ & Athlete's heart & Cardiac amyloidosis & Anderson-Fabry disease \\
\hline $\begin{array}{l}\text { Left } \\
\text { ventricle }\end{array}$ & $\begin{array}{l}\text { - Asymmetric LVH in } \\
\text { most cases }\end{array}$ & $\begin{array}{l}\text { - Moderate LVH } \\
\text { - Concentric }\end{array}$ & $\begin{array}{l}\text { - Mild to moderate LVH } \\
\text { - Concentric } \\
\text { - Enlarged LV }\end{array}$ & $\begin{array}{l}\text { - Moderate LVH } \\
\text { - Concentric } \\
\text { - Sparkly pattern }\end{array}$ & $\begin{array}{l}\text { Concentric LVH } \\
\text { Basal/inferolateral } \\
\text { hypokinesia }\end{array}$ \\
\hline $\begin{array}{l}\text { Right } \\
\text { ventricle }\end{array}$ & $\begin{array}{l}\text { - Increased free wall } \\
\text { thickness in some } \\
\text { cases }\end{array}$ & $\begin{array}{l}\text { - No specific } \\
\text { abnormalities }\end{array}$ & $\begin{array}{l}\text { - Physiological dilation } \\
\text { - Increased free wall } \\
\text { thickness }\end{array}$ & $\begin{array}{l}\text { - Increased free wall } \\
\text { thickness }\end{array}$ \\
\hline Atria & - Enlarged left atrium & $\begin{array}{l}\text { - Enlarged left atrium } \\
\text { in some cases }\end{array}$ & $\begin{array}{l}\text { - Normal or mildly } \\
\text { enlarged }\end{array}$ & $\begin{array}{l}\text { - Enlarged left atrium. } \\
\text { - Increased interatrial } \\
\text { septum thickness. }\end{array}$ & - Enlarged left atrium \\
\hline Valves & $\begin{array}{l}\text { - Leaflet } \\
\text { abnormalities } \\
\text { Excessive tissue, } \\
\text { prolapse, mismatch) }\end{array}$ & $\begin{array}{l}\text { - No specific } \\
\text { abnormalities }\end{array}$ & $\begin{array}{l}\text { - No specific } \\
\text { abnormalities } \\
\text { muscles and } \\
\text { atrioventricular cardiac } \\
\text { valves }\end{array}$ & cardiac valves \\
\hline $\begin{array}{l}\text { Other } \\
\text { features }\end{array}$ & $\begin{array}{l}\text { - Papillary muscles } \\
\text { abnormalities } \\
-30 \% \text { of patients } \\
\text { with SAM and LVOTO } \\
\text { at rest }\end{array}$ & $\begin{array}{l}\text { - Regression of LVH } \\
\text { after antihypertensive } \\
\text { treatment }\end{array}$ & $\begin{array}{l}\text { - LV thickness decreases } \\
\text { with deconditioning } \\
\text { - Apical sparing with } \\
\text { speckle-tracking analysis }\end{array}$ & - Binary sign (not specific) \\
\hline
\end{tabular}


Table 3. Echocardiographic preoperative assessment.

\begin{tabular}{|l|l|}
\hline \multicolumn{2}{|c|}{ Echocardiographic preoperative assessment } \\
\hline 1. Mechanism of obstruction. & - Systolic anterior motion (SAM). \\
& - Mid cavity due to mid-septal hypertrophy. \\
& - Sub-aortic membrane. \\
& - Aortic stenosis. \\
& - Abnormal papillary muscle insertion into the \\
& anterior mitral leaflet. \\
& - Accessory papillary muscles producing mid-cavity \\
obstruction. \\
- Enlarged mitral valve leaflets. \\
\hline of. Extension, width and depth & - Accessory mitral valve tissue. \\
\hline papillary muscles. & sistance from the point of anterior mitral leaflet- \\
mitral valve, annulus and & coronary cusp and downwards to the apex (Figure \\
3. Morphological and & 7C). \\
& - Location of the endocardial fibrous plaque. \\
& - Apical extent of the septal buldge. \\
\hline
\end{tabular}


Table 4: Ecocardiography versus CMR in HCM

\begin{tabular}{|c|c|c|}
\hline & Echocardiography & Cardiac magnetic resonance \\
\hline Advantages & $\begin{array}{l}\text { - Availability } \\
\text { - Uncomplicated evaluation of } \\
\text { dynamic features such as } \\
\text { left ventricular outflow tract } \\
\text { obstruction and systolic } \\
\text { anterior motion. } \\
\text { - Evaluation of diastolic } \\
\text { function. } \\
\text { Myocardial longitudinal } \\
\text { velocities and deformation } \\
\text { parameters useful in pre- } \\
\text { hypertrophic states. } \\
\text { - } \\
\text { Intraoperative studies } \\
\text { available to guide myectomy } \\
\text { or alcohol septal ablation. } \\
\text { screening. }\end{array}$ & 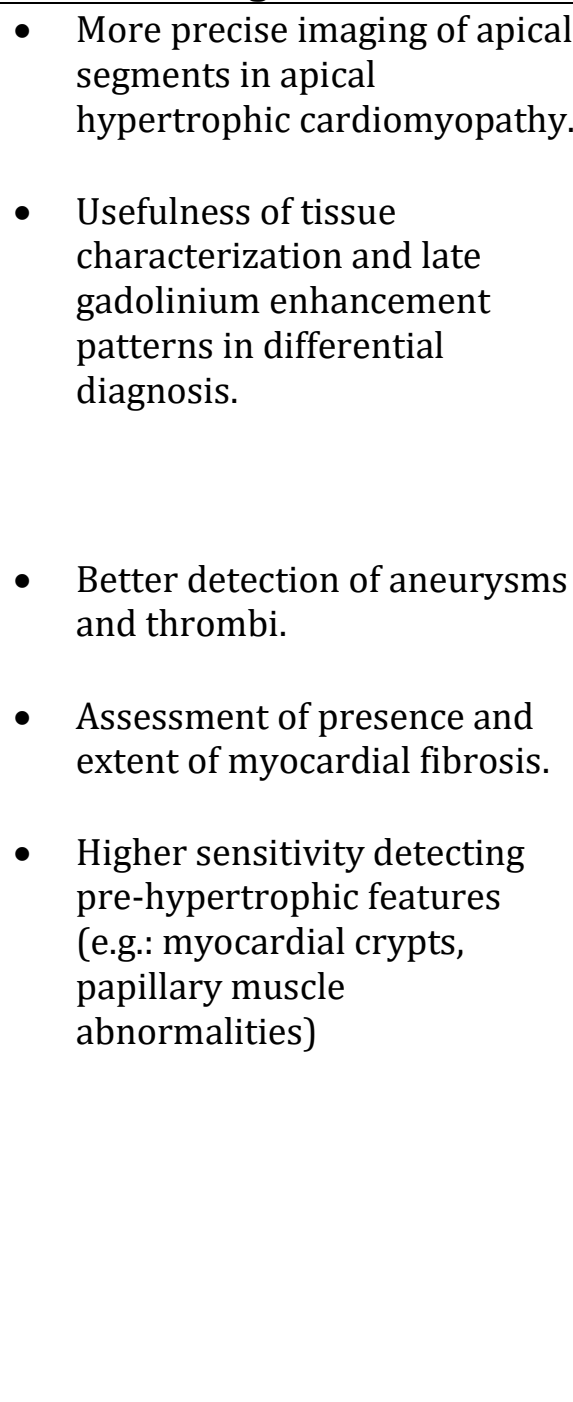 \\
\hline Disadvantages & $\begin{array}{l}\text { - } \begin{array}{l}\text { Limited issue } \\
\text { characterization. }\end{array} \\
\text { - Worse imaging of anterior } \\
\text { and apical segments. } \\
\text { - } \begin{array}{l}\text { No direct visualization of } \\
\text { myocardial fibrosis. }\end{array} \\
\text { - Acoustic window } \\
\text { dependency }\end{array}$ & $\begin{array}{l}\text { - Availability } \\
\text { - Evaluation of left ventricular } \\
\text { outflow tract obstruction and } \\
\text { systolic anterior motion is less } \\
\text { accessible and more } \\
\text { complicated as compared to } \\
\text { echocardiography. }\end{array}$ \\
\hline
\end{tabular}




\section{Supplementary Material Online}

\section{Video S1. Mid-ventricular obstruction.}

Transthoracic apical 4-chamber view from a patient with HCM and mainly apical and midventricular hypertrophy with mid-ventricular collapse during systole.

\section{Video S2. 3D intraoperative TOE.}

3D TOE showing moderate-to-severe eccentric and posterior MR in a patient undergoing myectomy.

Video S3. Colour Doppler intraoperative TOE showing moderate-to-severe mitral regurgitation in a patient undergoing myectomy.

Calcified mitral annulus and two jets, one central and one eccentric, suggesting a mixed aetiology, not optimal for mitral repair.

Video S4. Myocardial contrast transthoracic echocardiogram during ASA.

Apical 4-chamber view showing opacified area of VM-septum contact after contrast injection in the first septal vessel.

Video S5. Intraprocedural transhoratic echocardiogram evaluating ASA result.

Apical colour Doppler 5-chamber view after alcohol injection showing increased echogenicity in the septum, absence of SAM or LVOTO and residual mild MR. 